\title{
Bradicardia asociada a crisis epilépticas. Casos clínicos
}

\author{
Juan Idiáquez $\mathrm{C}^{1}$, Enzo Rivera $\mathrm{T}^{1}$, Julia Santin $\mathbf{M}^{2}$, \\ Jaime $\mathbf{G}$ odoy $\mathbf{F}^{2}$.
}

\section{Bradycardia during epileptic seizures. Report of two patients}

Cardiac rate and rhythm due to autonomic activation, may occur during epileptic seizures. They are probably a consequence of the spreading seizure activity to structures involved in the autonomic control of cardiac rhythm. Sinus tachycardia is the most frequent finding (90\%). Ictal bradycardia is rare and life threatening asystoles require cardiac pacemaker insertion. We report two patients with a right frontal seizure associated to bradycardia, during simultaneous EEG/ECG monitoring. Interictal autonomic function tests showed preserved sympathetic and cardiac vagal responses. Normal interictal autonomic function tests, suggested a transient failure of sympatho-vagal balance (Rev Méd Chile 2009; 137: 401-4).

(Key words: Autonomic nervous system; Bradycardia; Epilepsy)

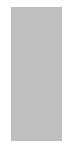

Recibido el 30 de mayo, 2008. Aceptado el 10 de octubre, 2008.

${ }^{1}$ Cátedra de Neurología, Facultad de Medicina, Universidad de Valparaíso. Valparaíso, Chile. ${ }^{2}$ Departamento de Neurología, Escuela de Medicina, Pontificia Universidad Católica de Chile. Santiago de Chile.

$\mathrm{D}$ urante las crisis epilépticas ocurren cambios en la función autonómica cardiaca. La corteza insular se considera el área más importante de la red autonómica central en el control de la regulación cardiovascular y tiene extensas conexiones con otros centros involucrados en el balance simpático-vagal ${ }^{1}$. La respuesta simpática predomina durante la mayoría de las crisis causando taquicardia ${ }^{1-3}$. Alteraciones electrocardiográficas asociadas a crisis epilépticas ocurren aproximadamente en $40 \%$, estos eventos en su mayoría son taquicardias sinusales (90\%), también ocurren despolarizaciones auriculares prematuras, complejos ventriculares prematuros, desnivel del segmento ST, bradicardias, pausas sinusales y asistolias ${ }^{3}$.

Correspondencia a: Juan Idiáquez C. 4 Norte \# 1093, Depto. 22, Viña del Mar. Fax: (56) 322975623.

E mail: idiaquez@123.cl
La ocurrencia de bradicardia ictal es rara (menos de 5\%) y en su mayoría se origina por activación de la corteza temporal ${ }^{2-5}$. Si bien se ha descrito una predominancia del hemisferio izquierdo, se han reportado casos de bradicardia por activación de hemisferio cerebral derecho y también bilateral ${ }^{5}$. La asistolia ocurre entre $0,3 \%$ y $2,4 \%$ de los pacientes con epilepsia refractaria y su detección es importante para prevenir eventos fatales, eventualmente con la instalación de marcapasos de demanda ${ }^{6}$. Se describen dos casos de bradicardia durante el monitoreo simultáneo de EEG/ECG de crisis epilépticas focales y el estudio de la función autonómica interictal.

\section{Casos ClínICOS}

Caso 1. Hombre de 34 años sin antecedentes de enfermedad sistémica ni de cardiopatía. Desde la 
edad de 21 años presentó crisis epilépticas parciales complejas refractarias. El examen físico general y neurológico fue normal. Durante años no fue posible controlar las crisis y presentaba un promedio de 2 crisis diarias. Recibió fenobarbital y fenitoína, cuyos niveles plasmáticos estaban en rango terapéutico. Repetidos estudios de electroencefalograma (EEG) ambulatorios mostraron actividad epiléptica frontal derecha. La resonancia magnética (RM) craneal mostró atrofia temporal derecha.

Un estudio de monitoreo video-EEG/ECG computarizado de $24 \mathrm{~h}$ mostró 15 crisis parciales complejas con focalización sobre región frontocentral derecha. Durante las crisis tuvo bradicardia de 44 latidos/min. El estudio autonómico interictal mostró una función simpática y cardiovagal normal (Tabla 1).

Caso 2. Niño de 9 años sin antecedentes de enfermedad sistémica ni cardiopatía, que a la edad de 3 años comenzó a presentar crisis epilépticas parciales complejas refractarias. El examen físico general y neurológico fue normal. Presentaba como promedio 2 crisis semanales, a pesar que tenía niveles plasmáticos de carbamazepina y lamotrigina en rango terapéutico. Los estudios de EEG ambulatorios mostraron actividad epiléptica frontal derecha. La RM craneal fue normal. Un estudio de monitoreo video-EEG/ECG computarizado mostró 3 crisis parciales complejas con focalización sobre región frontocentral derecha (Figura 1). Se registró bradicardia ictal en varias crisis, siendo la mayor de 36 latidos/min. El estudio autonómico interictal mostró respuestas simpáticas y parasimpáticas normales (Tabla 1).

\section{DisCUSIÓN}

En estos dos casos de bradicardia ictal, el registro electroencefalográfico en cuero cabelludo, mostró

\section{Tabla 1. Resultados de los exámenes de monitoreo EEG -EC G en dos casos de epilepsia parcial compleja y de la función interictal del sistema nervioso autonómico simpático y parasimpático}

\begin{tabular}{|c|c|c|}
\hline & Caso 1 & Caso 2 \\
\hline Edad (años) & 34 & 9 \\
\hline EEG ictal & $\begin{array}{l}\text { Espiga-onda lenta } \\
\text { frontocentral derecha }\end{array}$ & $\begin{array}{l}\text { Espigas en } \\
\text { región frontal } \\
\text { derecha }\end{array}$ \\
\hline \multicolumn{3}{|l|}{ Registro ECG* } \\
\hline FC basal & 73 & 62 \\
\hline FC ictal & 44 & 36 \\
\hline \% disminución FC & 40 & 42 \\
\hline \multicolumn{3}{|l|}{ Función autonómica interictal** } \\
\hline \multicolumn{3}{|l|}{ Simpática: } \\
\hline Caída postural presión arterial sistólica (mmHg) & 0 & 0 \\
\hline Ejercicio isométrico alza presión arterial diastólica (mmHg) & 14 & 16 \\
\hline \multicolumn{3}{|l|}{ Parasimpática: } \\
\hline Respiración máxima & 29 & 31 \\
\hline Índice de Valsalva & 1,6 & 1,5 \\
\hline Índice 30:15 & 1,1 & 1,4 \\
\hline
\end{tabular}

*Registro electrocardiográfico durante el monitoreo video-EEG, FC: frecuencia cardíaca (latidos/min). **Valores normales: caída postural presión arterial sistólica: $<20 \mathrm{mmHg}$. Ejercicio isométrico, alza presión arterial diastólica $\geq 12 \mathrm{mmHg}$. Respiración máxima (variación en latidos/min): $\geq 10$. Índice de Valsalva: menores de 60 años $\geq 1,5$. Índice 30:15: $\geq 1,1$ 


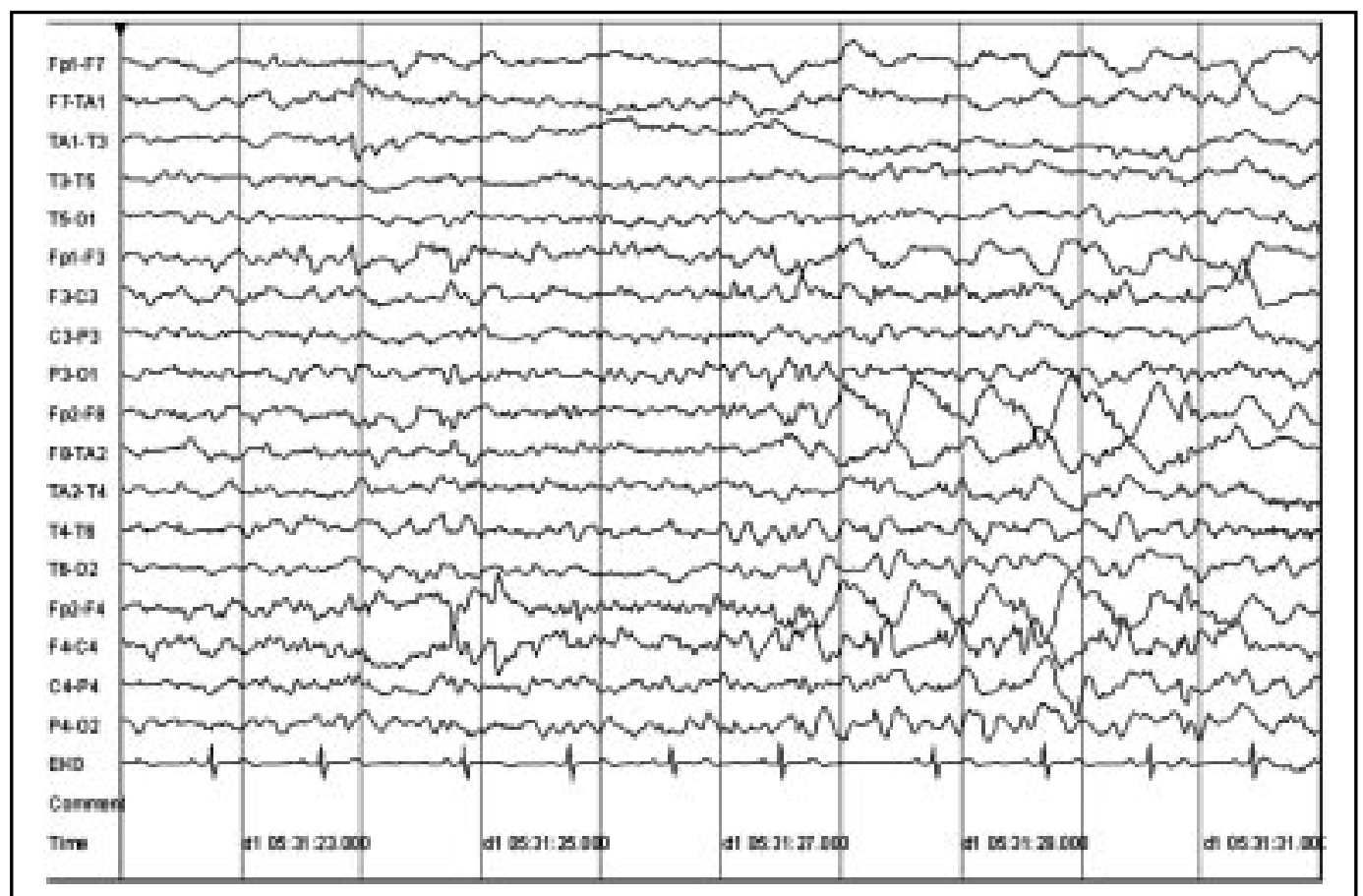

Figura 1. Registro simultáneo de EEG/ECG. En los canales 10 (Fp2-F8), 15 (Fp2-F8) y 16 (Fp2-F4), se muestra la actividad epileptiforme a nivel fronto-central del hemisferio cerebral derecho. El canal inferior (EKG) muestra el registro electrocardiográfico, en donde se observa que la bradicardia precede a la aparición de la descarga epiléptica.

un foco epiléptico frontal derecho. En el caso 1, la RMN mostró una atrofia temporal derecha. Respecto a la localización de la actividad epileptiforme que provoca una bradicardia ictal, la mayoría de los casos se asocia a un foco epiléptico en la corteza temporal 5 . Se ha planteado que la bradicardia ictal depende de una activación cortical bilateral $^{5}$. Es posible que un registro directo de la actividad eléctrica en la corteza cerebral, hubiera mostrado la actividad epiléptica, en la corteza temporal medial o insular.

En relación con el resultado de las pruebas de función autonómica en el periodo interictal, los dos pacientes tenían respuestas simpáticas normales de la presión arterial y también de la función vagal cardiaca. Estudios en pacientes con epilepsia del lóbulo temporal, durante el periodo interictal, han mostrado respuestas simpáticas conservadas de la presión arterial durante el cambio postural y de la activación parasimpática cardiaca, pero sí se ha encontrado una mala regulación de la frecuencia cardiaca con el cambio postural ${ }^{7}$. Se ha descrito en pacientes con epilepsia del lóbulo temporal, durante el periodo interictal, una disfunción a nivel de la inervación simpática del corazón, utilizando el método de ventriculografía isotópica, con metayodobenzilguanidina (MIBG) (análogo de la noradrenalina) $)^{8}$. Los trastornos de la frecuencia cardiaca ocurrirían por una alteración transitoria en el balance simpático-vagal, causada por la actividad epiléptica. A este respecto es importante señalar que en los casos de bradicardia ictal y de asistolia ocurre un predominio transitorio del tono vagal a nivel cardiaco. Una actividad cardioinhibitoria similar también ocurre durante el síncope vasovagal ${ }^{9}$, esto ha llevado a plantear que en ocasiones la asistolia ictal no necesariamente es grave, ya que posiblemente en estos casos al igual que en el síncope vasovagal, se activan mecanismos compensatorios que detienen la hiperactividad parasimpática, y se recupera así el balance simpático- vagal normal?. 
La presencia de asistolia durante una crisis epiléptica, se asocia a una pérdida del tono muscular, similar a la que ocurre durante el síncope vasovagal ${ }^{10,11}$. Posiblemente esta hipotonía muscular se asocia a una hipoperfusión cerebral secundaria a la asistolia.

También es importante considerar la acción potencial de los fármacos antiepilépticos sobre el ritmo cardiaco, el uso de carbamazepina oral se asocia a una disminución de las respuestas simpáticas y parasimpáticas a nivel cardiaco ${ }^{7,12}$. El uso de fenitoína sódica endovenosa puede provocar bradicardia ${ }^{7}$. Un paciente (caso 2) recibía carbamazepina oral, lo que podría haber influido en la génesis de la bradicardia observada. También es importante señalar que los pacientes no presentaban patología cardiaca primaria ni factores de riesgo cardiovascular.

El monitoreo EEG/ECG pesquisa la presencia de arritmias cardiacas y determina su gravedad, así la bradicardia, puede ser subclínica, como en

\section{REFERENCIAS}

1. Devinsky O. Effects of seizures on autonomic and cardiovascular function. Epilepsy Currents 2004; 4: 43-6.

2. Freeman R. Cardiovacular manifestations of autonomic epilepsy. Clin Auton Res 2006; 16: 12-7.

3. Ziljmans M, Flanagan D, Gotman J. Heart rate changes and ECG abnormalities during epileptic seizures: prevalence and definition of an objective clinical sign. Epilepsia 2002; 43: 847-54.

4. Tinuper P, Bisulli A, Cerillo A, Carcangiu R, Marini C, Pierangeli G, Cortelli P. Ictal bradycardia in partial epileptic seizures. Autonomic investigation in three cases and literature review. Brain 2001; 124: 236171.

5. Britton JW, Ghearing GR, Benarroch EE, Cascino GD. The Ictal Bradycardia Syndrome: localization and lateralization. Epilepsia 2006; 47: 737-44.

6. Britton JW, Benarroch EE. Seizures and syncope. Clin Auton Res 2006; 16: 18-28.

7. Ansakorpi H, Korpelainen JT, Suominen K, Tolonen U, MYlLYlä VV, IsOJÄRVI JIT. Interictal cardiovascular estos dos casos, pero también puede ser potencialmente grave. Se han reportado casos de pacientes epilépticos en los cuales se encontró asistolia ictal prolongada, lo que obligó a la implantación de marcapaso cardiaco. En dos casos de Britton et $\mathrm{al}^{6}$ y en cuatro casos de RuggGunn et $a^{13}$ se debió implantar un marcapaso. Además este monitoreo permite observar los eventuales efectos cardiacos adversos de los fármacos antiepilépticos que pueden sumarse a una bradicardia preexistente. Finalmente es importante señalar que los pacientes epilépticos pueden sufrir una muerte súbita (SUDEP) no traumática ni secundaria a causa médica obvia y en ausencia de status epiléptico. Un posible mecanismo de SUDEP es una arritmia cardiaca letal, debida a una brusca alteración en el balance simpático-vagal ${ }^{2,6}$, otras posibles causas son una injuria a la fibra muscular cardiaca por exceso de catecolamina, apneas centrales y edema pulmonar neurogéni$\mathrm{co}^{6}$.

autonomic responses in patient with temporal lobe epilepsy. Epilepsia 2000; 41: 42-7.

8. Druschky A, Hilz MJ, Hopp P, Platsch G, RadespielTröger M, Druschky K ET AL. Interictal cardiac autonomic dysfunction in temporal lobe epilepsy demonstrated by [(123)I] metaiodobenzylguanidineSPECT. Brain 2001; 124: 2372-82.

9. Schuele SU, Bermeo AC, Locatelli E, Burgess RC, LÜDERS HO. Ictal asystole: a benign condition? Epilepsia 2008; 49: 168-71.

10. Schuele SU, Bermeo AC, Alexopoulos AV, Locatelli ER, Burguess RC, Dinner DS, Foldvary-Schaefer N. Videoelectrographic and clinical features in patients with ictal asystole. Neurology 2007; 434-41.

11. Ghearing GR, Munger TM, Jaffe AS, Benarroch EE, BRITTON JW. Clinical cues for detecting ictal asystole. Clin Auton Res 2007; 7: 221-6.

12. Persson H, Ericson M, Tomson T. Carbamazepine affects autonomic cardiac control in patients with newly diagnosed epilepsy. Epilepsy Res 2003; 57: 69-75.

13. Rugg-Gunn FJ, Simister RJ, Squirrell M, Holdright DR, Duncan JS. Cardiac arrhytmias in focal epilepsy: a prospective long-term study. Lancet 2004; 364: 2212-19. 